\title{
Synthesis of sulfur-containing heterocyclic compounds by cyclo- condensation of acetylenic derivatives of anthraquinone with sodium sulfide
}

\author{
Mark S. Shvartsberg* and Irena D. Ivanchikova \\ Institute of Chemical Kinetics and Combustion, Siberian Branch of \\ the Russian Academy of Sciences, 630090 Novosibirsk, Russia \\ E-mail: shvarts@kinetics.nsc.ru
}

\section{Dedicated to Professor Boris A. Trofimov on the occasion of his $65^{\text {th }}$ birthday}

(received 03 June 03; accepted 09 July 03; published on the web 23 July 03)

\begin{abstract}
Reaction of vic- alkynylchloro- and vic- chloro-(1-oxoalk-2-ynyl)-anthraquinones with $\mathrm{Na}_{2} \mathrm{~S}$ in ethanol, with short heating, has been shown to afford anthrathiophenediones and anthrathiopyrantriones, respectively, generally in good yields. Under the same conditions, 1alkynylanthraquinones also undergo cyclocondensation to give anthra[2,1-b]thiophene-6,11diones.
\end{abstract}

Keywords: Acetylenic derivatives of anthraquinone, cyclocondensation, anthrathiophenediones, anthrathiopyrantriones

\section{Introduction}

Condensed heterocyclic derivatives of quinones are of interest as biologically active substances and technical materials. A variety of such nitrogen- and oxygen- containing heterocycles has been described. There is much less information about sulfur- containing heterocyclic quinoid compounds. At the same time, the introduction of sulfur-containing rings into the structure of compounds often determines their pharmacological properties, ${ }^{1-3}$ reduces side-effects of drugs, ${ }^{4}$ or improves the technical characteristics of materials. ${ }^{5,6}$ Thus, substituted benzo[b]thiophenes are estrogen-receptor modulators, thrombin inhibitors, anti-tumor and anti-inflammation agents. ${ }^{1,2,7,8}$ Some of them are currently in pharmaceutical use or development. ${ }^{7,8}$

In this connection, we considered it reasonable to study synthetic pathways to anthraquinones annelated by thiophene and other sulfur- containing rings. To our knowledge, general methods for the synthesis even of anthrathiophenediones have not been described. Earlier we reported the synthesis of a number of condensed $\mathrm{N}$ - and $\mathrm{O}$ - heterocyclic quinoid 
systems based on acetylenic derivatives of quinones as key precursors. ${ }^{9-18}$ One would expect that the same "acetylenic" approach will turn out to be fruitful for the construction of an anthrathiophene system as well.

Recently, a method for synthesis of substituted benzo[b]thiophenes by cyclization of orthomethylthio- and ortho- benzylthio(alkynyl)benzenes under the action of electrophilic agents was elaborated. ${ }^{1,19,20}$ However, its expansion to derivatives of anthraquinone is complicated by the limited availability of the corresponding sulfides. Other methods of formation of benzothiophenes from acetylenic precursors are not sufficiently general. ${ }^{21-24}$

\section{Results and Discussion}

In anthraquinone, a chlorine atom, irrespective of its position, possesses a high nucleofugal lability. ${ }^{25}$ The triple bond in acetylenic derivatives of quinones has an enhanced electrophilicity and readily adds nucleophiles. ${ }^{26}$ When a halogen atom and an acetylenic substituent are arranged in the same ring of anthraquinone, they mutually activate each other. We supposed that the above chemical peculiarities of these anthraquinone derivatives would make it possible to annelate the anthraquinone nuclei with a thiophene ring by using vic- alkynylchloroanthraquinones as key acetylenes and $\mathrm{Na}_{2} \mathrm{~S}$ as the simplest cyclizing agent.

Indeed, 2-alkynyl-1-chloroanthraquinones 1 a-e did react with an excess of $\mathrm{Na}_{2} \mathrm{~S}$ in $95 \%$ ethanol at reflux for 10-20 min to give anthra[1,2-b]thiophene-6,11-diones 2a-e in 57-90\% yields.<smiles>[R]#Cc1ccc2c(c1Cl)C(=O)c1ccccc1C2=O</smiles><smiles>[R]c1cc2ccc3c(c2s1)C(=O)c1ccccc1C3=O</smiles>
a: $\mathrm{R}=\mathrm{H}$
b: $\mathrm{R}=\mathrm{Ph}$
d: $\mathrm{R}=\mathrm{CH}(\mathrm{OH}) \mathrm{Pr}-i$
c: $\mathrm{R}=\mathrm{CMe} \mathrm{OH}_{2} \mathrm{OH}$
e: $\mathrm{R}=\mathrm{CH}_{2} \mathrm{OH}$

\section{Scheme 1}

The 1-alkynyl-2-chloroanthraquinones 3a-c bearing the halogen atom in position 2 were condensed with $\mathrm{Na}_{2} \mathrm{~S}$ in the same way as the chloroacetylenes $\mathbf{1}$, and under the same conditions. 

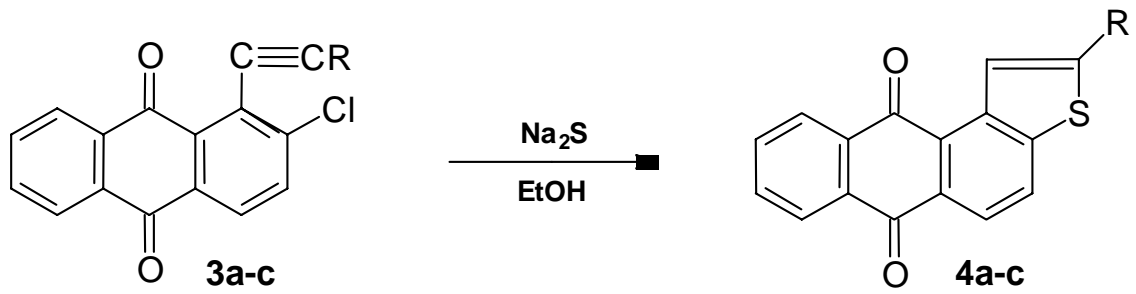
a: $\mathrm{R}=\mathrm{H}$
b: $\mathrm{R}=\mathrm{Ph}$
c: $\mathrm{R}=\mathrm{CMe}_{2} \mathrm{OH}$

\section{Scheme 2}

The yields of the anthra[2,1-b]thiophene-6,11-diones 4a-c were 66-97\%. It is noteworthy that in the ${ }^{1} \mathrm{H}$ NMR spectra of the anthrathiophenediones 4 the signal of the proton in the $\beta$ position of the thiophene ring, which is brought together spatially with the carbonyl group, is shifted to a lower field relative to that of the similar proton in spectra of compounds $2(\Delta \delta 1.3-$ $1.4 \mathrm{ppm})$.

Thus, the cyclocondensation of vic-alkynylchloroanthraquinones with $\mathrm{Na}_{2} \mathrm{~S}$ is a simple and convenient method for synthesis of angularly fused anthrathiophenediones.

We also applied the cyclocondensation under consideration to annelate anthraquinone with a thiopyran ring. In this case, the 1-chloro-2-(1-oxoalk-2-ynyl)anthraquinones $5 \mathbf{b}, \mathbf{c}, \mathbf{f}-\mathbf{h}$ and the 2chloro-3-(1-oxoalk-2-ynyl)anthraquinones $\mathbf{6 b}$,f were used as the acetylenic precursors. These compounds have, like the chloroacetylenes 1,3, the activated halogen atom and triple bond. Their condensation with $\mathrm{Na}_{2} \mathrm{~S}$ proceeds under the same conditions as that of compounds 1,3 and affords the anthra[1,2-b]thiopyran-4,7,12-triones $\mathbf{7 b , c , f - h}$ and the anthra[2,3-b]thiopyran-4,6,11triones 8b,f, respectively, in 68-94\% yields. It is essential to note that the possible competitive cyclization with closure to a 5 - membered ring is not observed.

The $4 H$ - anthra[1,2-b]pyran system provides the cyclic skeleton of the aglycones of the natural antitumor antibiotics of the kidamycin group and their biologically active analogs, which are obtained biochemically. ${ }^{27,28}$ The synthesized $\mathbf{7 b , c , f - h}$ are thio- analogs of these compounds.

In the synthesis of the kidamycin antibiotics there is a problem in the construction of the heterocycle bearing chemically sensitive substituents (alkenyl, epoxy, etc.). ${ }^{27}$ Our cyclocondensation, owing to its mild conditions, gives an opportunity to prepare anthrathiopyrantriones with such labile substituents. The preparation of the alkenylthiopyran $\mathbf{7 h}$ (a mixture of $Z$ - and $E$ - isomers) from the labile ketone $5 \mathbf{h}$, as well as of the cyclohexenylthiopyran $7 f$ demonstrates the applicability of the developed method for the synthesis of thio-analogs of aglycones of anthrapyrane antibiotics.

The initial step of the cyclocondensation of vic- acetylenic derivatives of chloroanthraquinones may be not only the substitution of the chlorine atom but also the addition of $\mathrm{Na}_{2} \mathrm{~S}$ to the triple bond. However, the intramolecular addition of $\mathrm{Na}_{2} \mathrm{~S}$, or other $\mathrm{S}$ - 
nucleophiles to alkynylquinones has not been studied. To confirm the possibility of a cyclocondensation pathway beginning with the addition of the cyclizing agent, we carried out the reaction of the 1-alkynylanthraquinones $\mathbf{9 b}, \mathbf{c}$ with $\mathrm{Na}_{2} \mathrm{~S}$ under the conditions of the cyclocondensation. The acetylenes $\mathbf{9 b}, \mathbf{c}$ were found to react with $\mathrm{Na}_{2} \mathrm{~S}$, but the reaction was not limited to the addition of this nucleophile, and was followed by cyclization of the primary adducts to result in the formation of anthra[2,1-b]thiophene-6,11-diones $\mathbf{4 b , \mathbf { b }}$ prepared before from the chloroacetylenes 3b,c. The cyclization step of the process seems to be an intramolecular nucleophilic oxidative substitution of the hydrogen atom, the quinone being the oxidant.<smiles>C#CC(=O)c1ccc2c(c1Cl)C(=O)c1ccccc1C2=O</smiles>

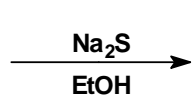<smiles>[R16]#CC(=O)c1cc2c(cc1Cl)C(=O)c1ccccc1C2=O</smiles>

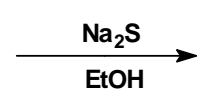

b: $R=P h$

c: $\mathrm{R}=\mathrm{CMe}_{2} \mathrm{OH}$

$\mathrm{f}: \mathrm{R}=\square$<smiles>[R]c1cc(=O)c2ccc3c(c2s1)C(=O)c1ccccc1C3=O</smiles><smiles>[R]c1cc(=O)c2cc3c(cc2s1)C(=O)c1ccccc1C3=O</smiles>

g: $\mathrm{R}=\mathrm{Bu}$

h: $\mathrm{R}=\mathrm{CMe}=\mathrm{CHMe}$

\section{Scheme 3}<smiles>[R]C#Cc1cccc2c1C(=O)c1ccccc1C2=O</smiles><smiles>CCOS(=O)(=O)OC</smiles><smiles>[R]c1cc2c3c(ccc2s1)C(=O)c1ccccc1C3=O</smiles>

b: $R=P h$

c: $\mathrm{R}=\mathrm{CMe}_{2} \mathrm{OH}$

\section{Scheme 4}


The yields of the anthrathiophenediones $4 \mathbf{b}, \mathbf{c}$ were $95 \%$. Our study of the scope and peculiarities of this reaction is in progress.

The key chloroacetylenes 1b-e and 3c were prepared by cross-coupling of 1-chloro-2-iodoanthraquinone $\mathbf{1 0}$ and 2-chloro-1-iodoanthraquinone 11 with the corresponding terminal acetylenes in aqueous dioxane in the presence of $\mathrm{Na}_{2} \mathrm{CO}_{3}, \mathrm{Pd}\left(\mathrm{PPh}_{3}\right)_{2} \mathrm{Cl}_{2}$ and $\mathrm{CuI}$ in $47-85 \%$ yields. We consider this cross-coupling procedure ${ }^{12,29}$ to be the best method for the introduction of acetylenic substituents into quinones. The chloroethynylanthraquinones 1a, 3a were synthesized from the tertiary acetylenic alcohols 1c, 3c by the retro- Favorsky reaction. ${ }^{29} 2$ Chloro-1-phenylethynylanthraquinone $\mathbf{3 b}$ was prepared as described earlier. ${ }^{26}$<smiles>[R]C#Cc1ccc2c(c1Cl)C(=O)c1ccccc1C2=O</smiles><smiles></smiles>
a: $\mathrm{R}=\mathrm{H}$
b: $\mathrm{R}=\mathrm{Ph}$
c: $\mathrm{R}=\mathrm{CMe}{ }_{2} \mathrm{OH}$
d: $\mathrm{R}=\mathrm{CH}(\mathrm{OH}) \mathrm{Pr}-i$
e: $\mathrm{R}=\mathrm{CH}_{2} \mathrm{OH}$

\section{Scheme 5}

The acetylenic ketones $\mathbf{5 b , c , f - h}$ and $\mathbf{6 b}, \mathbf{f}$ were synthesized by acylation of terminal acetylenes with 1-chloroanthraquinonoyl 2-chloride 12 and 2-chloroanthraquinonoyl 3-chloride 13, respectively, in a system of $\mathrm{NEt}_{3}-\mathrm{Pd}\left(\mathrm{PPh}_{3}\right)_{2} \mathrm{Cl}_{2}-\mathrm{CuI}-$-benzene ${ }^{30}$ in $49-76 \%$ yields. For the preparation of the vinylacetylenic ketone $\mathbf{5 h}$, a mixture of geometric isomers of 3-methylpent-3en-1-yne containing $\approx 70 \%$ of $\mathrm{Z}$ - isomer was used.

In summary, we have shown that condensation of vic- acetylenic derivatives of chloroanthraquinones with $\mathrm{Na}_{2} \mathrm{~S}$ offers a synthetic pathway to anthraquinones annelated by thiophene and thiopyran rings. 


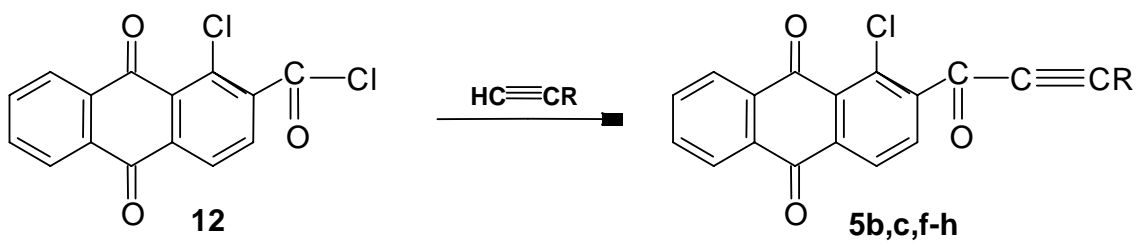<smiles>O=C(Cl)c1cc2c(cc1Cl)C(=O)c1ccccc1C2=O</smiles><smiles>[R]#CC(=O)c1cc2c(cc1Cl)C(=O)c1ccccc1C2=O</smiles>

\section{Scheme 6}

\section{Experimental Section}

\section{General procedure for the preparation of vic-alkynyl-chloroanthraquinones}

1-Chloro-2-phenylethynylanthraquinone (1b). 1-Chloro-2-iodoanthraquinone 10 (1.90 g, 5.1 $\mathrm{mmol}$ ) was dissolved in $60 \mathrm{~mL}$ of dioxane at $60-70^{\circ} \mathrm{C}$ under an atmosphere of $\mathrm{Ar}$, and then were added successively phenylacetylene $(0.80 \mathrm{~g}, 0.85 \mathrm{mmol}), \mathrm{Pd}\left(\mathrm{PPh}_{3}\right)_{2} \mathrm{Cl}_{2}(0.040 \mathrm{~g}), \mathrm{CuI}(0.040 \mathrm{~g})$ and $30 \mathrm{~mL}$ of aqueous solution of $\mathrm{Na}_{2} \mathrm{CO}_{3}(0.70 \mathrm{~g}, 6.6 \mathrm{mmol})$ heated beforehand to $70-80^{\circ} \mathrm{C}$. The mixture was heated at reflux with stirring for $15 \mathrm{~min}$. After complete consumption of the starting iodide 10 (monitoring by TLC), the mixture was cooled, diluted with $200 \mathrm{~mL}$ of $\mathrm{CHCl}_{3}$ and washed with water. The solvent was evaporated under reduced pressure, the residue purified by flash chromatography on silica gel using toluene as the eluent, and recrystallized from toluene-hexane to give 1-chloro-2-phenylethynylanthraquinone $\mathbf{1 b}$ (1.50 g, 85 \%), m.p. 193.5$194^{\circ} \mathrm{C}^{26}$

1-Chloro-2-(3-hydroxy-3-methylbutynyl)anthraquinone (1c). The cross-coupling was carried out by the general procedure with 1-chloro-2-iodoanthraquinone 10 (2.50 g, $6.8 \mathrm{mmol})$ and 3methylbut-1-yn-3-ol $(0.72 \mathrm{~g}, 0.85 \mathrm{mmol})$ in $100 \mathrm{~mL}$ of dioxane and $50 \mathrm{~mL}$ of water in the presence of $\mathrm{Na}_{2} \mathrm{CO}_{3}(0.72 \mathrm{~g}, 6.8 \mathrm{mmol}), \mathrm{Pd}\left(\mathrm{PPh}_{3}\right)_{2} \mathrm{Cl}_{2}(0.050 \mathrm{~g})$, and $\mathrm{CuI}(0.025 \mathrm{~g})$. The crude product was chromatographed on silica gel in a benzene-ether mixture to yield $1.84 \mathrm{~g}(84 \%)$ of 1-chloro-2-(3-hydroxy-3-methylbutynyl)anthraquinone 1c; ${ }^{17}$ m.p. $168-168.5^{\circ} \mathrm{C}$; IR $\left(\mathrm{CHCl}_{3}\right)$, $v_{\text {max }}$ : $1690(\mathrm{C}=\mathrm{O}), 2240(\mathrm{C} \equiv \mathrm{C}), 3605(\mathrm{OH}) \mathrm{cm}^{-1} ;{ }^{1} \mathrm{H} \mathrm{NMR}\left(\mathrm{CDCl}_{3}, 200 \mathrm{MHz}\right) \delta 1.70(\mathrm{~s}, 6 \mathrm{H}$, Me), 7.83 (d, 1H, $J=8.1 \mathrm{~Hz}, \mathrm{H}-3), 7.75-7.90$ (m, 2H, H-6,7), 8.26 (d, 1H, J = 8.1 Hz, H-4), 8.20-8.35 (m, 2H, H-5,8); Anal. Calcd for $\mathrm{C}_{19} \mathrm{H}_{13} \mathrm{O}_{3} \mathrm{Cl}$ : C, 70.27; H, 4.03; Cl, 10.91. Found: C, 70.40; H, 3.95; Cl, 10.78\%. 
1-Chloro-2-(4-methyl-3-hydroxypentynyl)anthraquinone (1d). The cross-coupling was achieved by the general procedure with 1-chloro-2-iodoanthraquinone $\mathbf{1 0}$ (2.00 g, $5.4 \mathrm{mmol})$ and 4-methylpent-1-yn-3-ol (1.05 g, $10.8 \mathrm{mmol})$ in $80 \mathrm{~mL}$ of dioxane and $40 \mathrm{~mL}$ of water in the presence of $\mathrm{Na}_{2} \mathrm{CO}_{3}(1.00 \mathrm{~g}, 9.4 \mathrm{mmol}), \mathrm{Pd}\left(\mathrm{PPh}_{3}\right)_{2} \mathrm{Cl}_{2}(0.060 \mathrm{~g})$, and $\mathrm{CuI}(0.045 \mathrm{~g})$ for $5 \mathrm{~min}$. The yield of 1-chloro-2-(4-methyl-3-hydroxypentynyl)anthraquinone $\mathbf{1 d}^{17}$ was $0.87 \mathrm{~g}$ (47\%); m.p. $160-161.5^{\circ} \mathrm{C}$; IR $\left(\mathrm{CHCl}_{3}\right)$, v $\mathrm{vax}_{\max }$ : $1690(\mathrm{C}=\mathrm{O}), 2235(\mathrm{C} \equiv \mathrm{C}), 3400$ br., $3615(\mathrm{OH}) \mathrm{cm}^{-1} ;{ }^{1} \mathrm{H}$ NMR $\left(\mathrm{CDCl}_{3}, 200 \mathrm{MHz}\right) \delta 1.10(\mathrm{~d}, 3 \mathrm{H}, J=6.7 \mathrm{~Hz}, \mathrm{Me}), 1.13(\mathrm{~d}, 3 \mathrm{H}, J=6.7 \mathrm{~Hz}, \mathrm{Me}), 1.95-2.10$ (m, 2H, $\underline{\mathrm{CHMe}} 2, \mathrm{OH}), 4.45-4.55(\mathrm{~m}, 1 \mathrm{H}, \mathrm{CHO}), 7.75-7.85$ (m, 2H, H-6,7), 7.83 (d, 1H, J=8.0 $\mathrm{Hz}, \mathrm{H}-3), 8.25$ (d, $1 \mathrm{H}, J=8.0 \mathrm{~Hz}, \mathrm{H}-4), 8.25-8.35$ (m, $2 \mathrm{H}, \mathrm{H}-5,8)$; Anal. Calcd for $\mathrm{C}_{20} \mathrm{H}_{15} \mathrm{O}_{3} \mathrm{Cl}$ : C, 70.90; H, 4.46; Cl, 10.46. Found: C, 70.78; H, 4.46; Cl, 10.40\%.

1-Chloro-2-(3-hydroxypropynyl)anthraquinone (1e). The reaction was carried out by the general procedure with 1-chloro-2-iodoanthraquinone $10(0.74 \mathrm{~g}, 2.0 \mathrm{mmol})$ and propargyl alcohol $(0.20 \mathrm{~g}, 3.57 \mathrm{mmol})$. The crude product in toluene was filtered through a layer of silica gel $(2-3 \mathrm{~cm})$ and crystallized from a toluene-hexane mixture to give 1-chloro-2-(3hydroxypropynyl)anthraquinone $1 \mathrm{e}^{17}(0.28 \mathrm{~g}, 47 \%)$; m.p. $197.5-199^{\circ} \mathrm{C}$; IR $\left(\mathrm{CHCl}_{3}\right), v_{\max }: 1690$ $(\mathrm{C}=\mathrm{O}), 2240(\mathrm{C} \equiv \mathrm{C}), 3400$ br., $3620(\mathrm{OH}) \mathrm{cm}^{-1} ;{ }^{1} \mathrm{H} \mathrm{NMR}\left(\mathrm{CDCl}_{3}, 200 \mathrm{MHz}\right) \delta 4.67\left(\mathrm{~s}, 2 \mathrm{H}, \mathrm{CH}_{2}\right)$, 7.75-7.90 (m, 3H, H-3,6,7), 8.20-8.30 (m, 3H, H-4,5,8); Anal. Calcd for $\mathrm{C}_{17} \mathrm{H}_{9} \mathrm{O}_{3} \mathrm{Cl}$ : C, 68.82; H, 3.06; Cl, 11.95. Found: C, 68.66; H, 3.02; Cl, 11.79\%.

2-Chloro-1-(3-hydroxy-3-methylbutynyl)anthraquinone (3c). The experiment was carried out by the general procedure with $1.80 \mathrm{~g}(4.9 \mathrm{mmol})$ of 2-chloro-1-iodo-anthraquinone 11 and $0.56 \mathrm{~g}$ (6.7 mmol) of 3-methylbut-1-yn-3-ol in $60 \mathrm{~mL}$ of dioxane and $30 \mathrm{~mL}$ of water in the presence of $0.32 \mathrm{~g}$ ( $3.0 \mathrm{mmol})$ of $\mathrm{Na}_{2} \mathrm{CO}_{3}, 0.030 \mathrm{~g}$ of $\mathrm{Pd}\left(\mathrm{PPh}_{3}\right)_{2} \mathrm{Cl}_{2}, 0.015 \mathrm{~g}$ of $\mathrm{CuI}$. The crude product was purified by chromatography on silica gel using $\mathrm{CHCl}_{3}$ as the eluent and recrystallized from a toluene-hexane mixture to yield $1.25 \mathrm{~g}$ (79 \%) of 2-chloro-1-(3-hydroxy-3-methylbutynyl)anthraquinone 3c; m.p. 183.5-184.5 ${ }^{\circ}$; IR $\left(\mathrm{CHCl}_{3}\right), v_{\max }$ : $1690(\mathrm{C}=\mathrm{O}), 2240(\mathrm{C} \equiv \mathrm{C}), 3400$ br., $3615(\mathrm{OH}) \mathrm{cm}^{-1} ;{ }^{1} \mathrm{H} \mathrm{NMR}\left(\mathrm{CDCl}_{3}, 200 \mathrm{MHz}\right) \delta 1.76(\mathrm{~s}, 6 \mathrm{H}, \mathrm{Me}), 2.97$ (brs, 1H, OH), 7.70-7.80 (m, 2H, H-6,7), 7.80 (d, 1H, J=8.5 Hz, H-3), 8.20-8.30 (m, 2H, H-5,8), 8.20 (d, 1H, J = 8.5 Hz, H-4); Anal. Calcd for $\mathrm{C}_{19} \mathrm{H}_{13} \mathrm{O}_{3} \mathrm{Cl}$ : C, 70.27; H, 4.03; Cl, 10.92. Found: C, 70.20; H, 4.04; Cl, $11.03 \%$.

1-Chloro-2-ethynylanthraquinone (1a). 1-Chloro-2-(3-hydroxy-3-methylbutynyl)anthraquinone 1c $(1.45 \mathrm{~g}, 4.5 \mathrm{mmol})$ and powdered $\mathrm{KOH}(1.00 \mathrm{~g}, 17.9 \mathrm{mmol})$ in $100 \mathrm{~mL}$ of dry benzene were stirred at $80^{\circ} \mathrm{C}$ for $45 \mathrm{~min}$ and filtered. The product was isolated by column chromatography on $\mathrm{Al}_{2} \mathrm{O}_{3}$ using toluene as the eluent and crystallized from a toluene-hexane mixture. The yield of 1-chloro-2-ethynylanthraquinone 1a was $0.90 \mathrm{~g}(73 \%)$; m.p. $211-212^{\circ} \mathrm{C}$; IR $\left(\mathrm{CHCl}_{3}\right), v_{\max }$ : $1690(\mathrm{C}=\mathrm{O}), 2130(\mathrm{C} \equiv \mathrm{C}), 3310(\mathrm{C} \equiv \mathrm{CH}) \mathrm{cm}^{-1} ;{ }^{1} \mathrm{H} \mathrm{NMR}\left(\mathrm{CDCl}_{3}, 200 \mathrm{MHz}\right) \delta 3.67(\mathrm{~s}, 1 \mathrm{H}$, $\mathrm{HC} \equiv \mathrm{C}), 7.75-7.90(\mathrm{~m}, 2 \mathrm{H}, \mathrm{H}-6,7), 7.91(\mathrm{~d}, 1 \mathrm{H}, J=8.1 \mathrm{~Hz}, \mathrm{H}-3), 8.20-8.35$ (m, 2H, H-5,8), 8.28 (d, $1 \mathrm{H}, J=8.1 \mathrm{~Hz}, \mathrm{H}-4)$; Anal. Calcd for $\mathrm{C}_{16} \mathrm{H}_{7} \mathrm{O}_{2} \mathrm{Cl}: \mathrm{C}, 72.06 ; \mathrm{H}, 2.65 ; \mathrm{Cl}, 13.29$. Found: C, 71.98; H, 2.84; Cl, 13.09\%.

2-Chloro-1-ethynylanthraquinone (3a). The retro-Favorsky reaction with 2-chloro-1-(3hydroxy-3-methylbutynyl)anthraquinone 3c (1.83 g, $5.6 \mathrm{mmol})$ and powdered $\mathrm{KOH}(0.97 \mathrm{~g}$, 
$17.3 \mathrm{mmol})$ in $200 \mathrm{~mL}$ of benzene $\left(80^{\circ} \mathrm{C}, 20 \mathrm{~min}\right)$, isolation and purification of the product were carried out as described for 1a, to give 2-chloro-1-ethynylanthraquinone 3a (0.47 g, 31 \%); m.p. 244-245 ${ }^{\circ}$; IR $\left(\mathrm{CHCl}_{3}\right), v_{\max }$ : $1690(\mathrm{C}=\mathrm{O}), 2120(\mathrm{C} \equiv \mathrm{C}), 3320(\mathrm{C} \equiv \mathrm{CH}) \mathrm{cm}^{-1} ;{ }^{1} \mathrm{H} \mathrm{NMR}\left(\mathrm{CDCl}_{3}\right.$, $200 \mathrm{MHz}) \delta 3.97$ (s, 1H, HC $\equiv \mathrm{C}), 7.70-7.90$ (m, 2H, H-6,7), 7.87 (d, 1H, J=8.4 Hz, H-3), 8.258.45 (m, 2H, H-5,8), 8.27 (d, $1 \mathrm{H}, J=8.4 \mathrm{~Hz}, \mathrm{H}-4)$; Anal. Calcd for $\mathrm{C}_{16} \mathrm{H}_{7} \mathrm{O}_{2} \mathrm{Cl}$ : C, 72.06; $\mathrm{H}$, 2.65; Cl, 13.29. Found: C, 72.18; H, 2.59; Cl, 13.26\%.

\section{General procedure for acylation}

1-Chloro-2-(1-oxo-3-phenylpropynyl)anthraquinone $\quad \mathbf{( 5 b ) . ~ T o ~ a ~ s o l u t i o n ~ o f ~ 1 - ~}$ chloroanthraquinonoyl-2- chloride $121.00 \mathrm{~g}$ (3.3 $\mathrm{mmol})$, prepared by refluxing 1chloroanthraquinone-2-carboxylic acid with $\mathrm{SOCl}_{2}$ for $4 \mathrm{~h}$, in $50 \mathrm{~mL}$ of dry benzene under Ar, were added $\mathrm{Et}_{3} \mathrm{~N}(1.1 \mathrm{~g}, 11.0 \mathrm{mmol}), \mathrm{Pd}\left(\mathrm{PPh}_{3}\right)_{2} \mathrm{Cl}_{2}(0.040 \mathrm{~g}), \mathrm{CuI}(0.040 \mathrm{~g})$ and phenylacetylene $(0.56 \mathrm{~g}, 5.5 \mathrm{mmol})$. The reaction mixture was stirred at r.t. for $20 \mathrm{~min}$, diluted with $300 \mathrm{~mL}$ of $\mathrm{CHCl}_{3}$, washed with water, dried over $\mathrm{MgSO}_{4}$ and evaporated under reduced pressure. The crude product was purified by flash chromatography on silica gel in toluene and crystallized from a toluene-hexane mixture to yield $0.85 \mathrm{~g} \quad(70 \%)$ of 1-chloro-2-(1-oxo-3phenylpropynyl)anthraquinone 5b; m.p. $183-183.5^{\circ} \mathrm{C}$; IR $\left(\mathrm{CHCl}_{3}\right), v_{\max }$ : 1670, $1690(\mathrm{C}=\mathrm{O})$, $2205(\mathrm{C} \equiv \mathrm{C}) \mathrm{cm}^{-1} ;{ }^{1} \mathrm{H}$ NMR $\left(\mathrm{CDCl}_{3}, 200 \mathrm{MHz}\right) \delta 7.35-7.55(\mathrm{~m}, 3 \mathrm{H}, 3 \mathrm{HPh}), 7.60-7.70(\mathrm{~m}, 2 \mathrm{H}$, 2HPh), 7.75-7.90 (m, 2H, H-6,7), 8.05 (d, 1H, J = 7.9 Hz, H-3), 8.25-8.35 (m, 2H, H-5,8), 8.41 (d, $1 \mathrm{H}, J=7.9 \mathrm{~Hz}, \mathrm{H}-4)$; Anal. Calcd for $\mathrm{C}_{23} \mathrm{H}_{11} \mathrm{O}_{3} \mathrm{Cl}: \mathrm{C}, 74.50 ; \mathrm{H}, 2.99 ; \mathrm{Cl}, 9.56$. Found: C, 74.48; H, 3.14; Cl, 9.65\%.

1-Chloro-2-(4-hydroxy-4-methyl-1-oxo-pentynyl)anthraquinone (5c). The reaction of 1chloro- anthraquinonoyl-2 chloride $12(0.37 \mathrm{~g}, 1.2 \mathrm{mmol})$ with 3-methylbut-1-yn-3-ol (0.22 g, 2.6 $\mathrm{mmol})$ in $25 \mathrm{~mL}$ of benzene in the presence of $\mathrm{Et}_{3} \mathrm{~N}(0.36 \mathrm{~g}, 3.6 \mathrm{mmol}), \mathrm{Pd}\left(\mathrm{PPh}_{3}\right)_{2} \mathrm{Cl}_{2}(0.015 \mathrm{~g})$, $\mathrm{CuI}(0.015 \mathrm{~g})$ was carried out by the general procedure to afford 1-chloro-2-(4-hydroxy-4methyl-1-oxo-pentynyl)anthraquinone 5c $(0.28 \mathrm{~g}, 65 \%)$; m.p. $145-146.5^{\circ} \mathrm{C}$; IR $\left(\mathrm{CHCl}_{3}\right), v_{\max }$ : 1670, $1690(\mathrm{C}=\mathrm{O}), 2225(\mathrm{C} \equiv \mathrm{C}), 3400$ br., $3610(\mathrm{OH}) \mathrm{cm}^{-1} ;{ }^{1} \mathrm{H}$ NMR $\left(\mathrm{CDCl}_{3}, 200 \mathrm{MHz}\right) \delta 1.62$ (s, 6H, Me), 2.20 (brs, 1H, OH), 7.75-7.85 (m, 2H, H-6,7), 7.94 (d, 1H, J=8.0 Hz, H-3), 8.208.30 (m, 2H, H-5,8), 8.35 (d, $1 \mathrm{H}, J=8.0 \mathrm{~Hz}, \mathrm{H}-4)$; Anal. Calcd for $\mathrm{C}_{20} \mathrm{H}_{13} \mathrm{O}_{4} \mathrm{Cl}$ : C, 68.09; $\mathrm{H}$, $3.71 ; \mathrm{Cl}, 10.05$. Found: C, 68.23; H, 3.89; Cl, 10.08\%.

1-Chloro-2-[3-(1-cyclohexenyl)-1-oxopropynyl]anthraquinone (5f). The experiment was carried out by the general procedure with 1-chloroanthraquinonoyl-2 chloride 12 (1.50 g, 4.9 $\mathrm{mmol})$ and 1-ethynylcyclohexene $(0.93 \mathrm{~g}, 8.8 \mathrm{mmol})$ in $70 \mathrm{~mL}$ of benzene in the presence of $\mathrm{Et}_{3} \mathrm{~N}(1.6 \mathrm{~g}, 15.7 \mathrm{mmol}), \mathrm{Pd}\left(\mathrm{PPh}_{3}\right)_{2} \mathrm{Cl}_{2}(0.050 \mathrm{~g}), \mathrm{CuI}(0.050 \mathrm{~g})$. The yield of 1-chloro-2-[3-(1cyclohexenyl)-1-oxopropynyl]anthraquinone 5 f was $1.15 \mathrm{~g}(63 \%)$; m.p. $168-169^{\circ} \mathrm{C}$; IR $\left(\mathrm{CHCl}_{3}\right)$, $v_{\text {max }}: 1670,1690(\mathrm{C}=\mathrm{O}), 2195(\mathrm{C} \equiv \mathrm{C}) \mathrm{cm}^{-1} ;{ }^{1} \mathrm{H} \mathrm{NMR}\left(\mathrm{CDCl}_{3}, 200 \mathrm{MHz}\right) \delta 1.45-1.75\left(\mathrm{~m}, 4 \mathrm{H}, 4^{\prime}\right.$, 5'-CH ), 2.10-2.30 (m, 4H, 3', 6'- $\left.\mathrm{CH}_{2}\right), 6.55-6.60$ (m, 1H, 2'-CH), 7.75-7.90 (m, 2H, H-6,7), 7.95 (d, 1H, $J=8.0 \mathrm{~Hz}, \mathrm{H}-3), 8.20-8.35$ (m, 2H, H-5,8), 8.36 (d, 1H, J = 8.0 Hz, H-4); Anal. Calcd for $\mathrm{C}_{23} \mathrm{H}_{15} \mathrm{O}_{3} \mathrm{Cl}$ : C, 73.70; H, 4.03; Cl, 9.46. Found: C, 73.80; H, 4.19; Cl, 9.61\%. 
1-Chloro-2-(1-oxohept-2-ynyl)anthraquinone (5g). The acylation was performed by the general procedure with 1-chloroanthraquinonoyl-2 chloride $12(0.40 \mathrm{~g}, 1.3 \mathrm{mmol})$ and 1-hexyne $(0.18 \mathrm{~g}, 2.2 \mathrm{mmol})$ to result in 1-chloro-2-(1-oxohept-2-ynyl)anthraquinone $5 \mathrm{~g}(0.30 \mathrm{~g}, 65 \%)$;

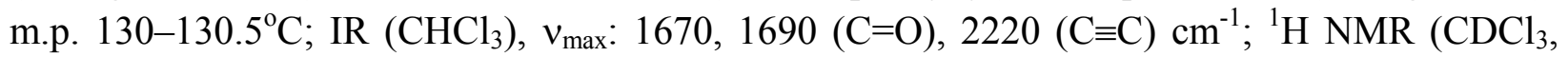
$200 \mathrm{MHz}) \delta 0.92(\mathrm{t}, 3 \mathrm{H}, J=7.2 \mathrm{~Hz}, \mathrm{Me}), 1.40-1.65\left(\mathrm{~m}, 4 \mathrm{H}, \beta-, \gamma-\mathrm{CH}_{2}\right), 2.47(\mathrm{t}, 2 \mathrm{H}, J=7.0 \mathrm{~Hz}$, $\left.\alpha-\mathrm{CH}_{2}\right), 7.75-7.85$ (m, 2H, H-6,7), 7.94 (d, 1H, J = 8.0 Hz, H-3), 8.20-8.30 (m, 2H, H-5,8), 8.36 $(\mathrm{d}, 1 \mathrm{H}, J=8.0 \mathrm{~Hz}, \mathrm{H}-4)$; Anal. Calcd for $\mathrm{C}_{21} \mathrm{H}_{15} \mathrm{O}_{3} \mathrm{Cl}$ : C, 71.90; H, 4.31; Cl, 10.11. Found: C, 71.68; H, 4.22; Cl, 10.19\%.

1-Chloro-2-(4-methyl-1-oxohex-4-en-2-ynyl)anthraquinone $\mathbf{( 5 h ) . ~ T h e ~ r e a c t i o n ~ o f ~ 1 - ~}$ chloroanthra- quinonoyl-2 chloride $12(1.40 \mathrm{~g}, 4.6 \mathrm{mmol})$ with a mixture of $Z$ - and $E$-isomers of 3-methylpent-3-en-1-yne containing $\approx 70 \%$ of Z-isomer $(0.74 \mathrm{~g}, 9.2 \mathrm{mmol})$ in $135 \mathrm{~mL}$ of benzene in the presence of $\mathrm{Et}_{3} \mathrm{~N}(1.3 \mathrm{~g}, 12.8 \mathrm{mmol}), \mathrm{Pd}\left(\mathrm{PPh}_{3}\right)_{2} \mathrm{Cl}_{2}(0.065 \mathrm{~g})$, and $\mathrm{CuI}(0.065 \mathrm{~g})$, was carried out for $10 \mathrm{~min}$ by the general procedure. The reaction mixture was filtered through a layer of silica gel, concentrated under reduced pressure, chromatographed on silica gel using toluene as the eluent and crystallized from a hexane-ether mixture to give 1-chloro-2-(4-methyl1-oxo-hex-4-en-2-ynyl)anthraquinone $5 \mathbf{h}(0.84 \mathrm{~g}, 52 \%)$; which was not sufficiently stable, and was used without further purification; ${ }^{1} \mathrm{H} \mathrm{NMR}\left(\mathrm{CDCl}_{3}, 200 \mathrm{MHz}\right) \delta 1.75(\mathrm{~d}, 3 \mathrm{H}, J=7.0 \mathrm{~Hz}$, $\underline{\mathrm{MeCH}}=\mathrm{C}$ E-isomer), $1.87\left(\mathrm{~d}, 3 \mathrm{H}, J=7.0 \mathrm{~Hz}, \underline{\mathrm{MeCH}}=\mathrm{C} Z\right.$-isomer), 1.92 (brs, $3 \mathrm{H}, \mathrm{CH}_{3}-\mathrm{C}=\mathrm{C} Z$ Zisomer), 1.95 (brs, $3 \mathrm{H}, \mathrm{CH}_{3}-\mathrm{C}=\mathrm{C}$ E-isomer), 6.10-6.20 (m, 1H, CH=C Z-isomer), 6.35-6.45 (m, 1H, CH=C E-isomer), 7.75-7.90 (m, 2H, H-6,7), 7.95 (d, 1H, J = 8.0 Hz, H-3 E-isomer), 7.98 (d, 1H, $J=8.0$ Hz, H-3 Z-isomer), 8.20-8.35 (m, 2H, H-5,8), 8.37 (d, 1H, J = 8.0 Hz, H-4 E-isomer), 8.38 (d, 1H, $J=8.0 \mathrm{~Hz}, \mathrm{H}-4 \mathrm{Z}$-isomer).

2-Chloro-3-(1-oxo-3-phenylpropynyl)anthraquinone (6b). Phenylacetylene $(0.43$ g, 4.2 mmol) was acylated by 2-chloroanthraquinonoyl-3 chloride 13 (0.65 g, $2.1 \mathrm{mmol})$, prepared by refluxing 2-chloroanthraquinone-3-carboxylic acid with $\mathrm{SOCl}_{2}$ for $4 \mathrm{~h}$, in $40 \mathrm{~mL}$ of dry benzene in the presence of $\mathrm{Et}_{3} \mathrm{~N}(0.72 \mathrm{~g}, 7.1 \mathrm{mmol}), \mathrm{Pd}\left(\mathrm{PPh}_{3}\right)_{2} \mathrm{Cl}_{2}(0.020 \mathrm{~g})$, and $\mathrm{CuI}(0.020 \mathrm{~g})$ by the general procedure. The reaction mixture was diluted with hexane $(60 \mathrm{~mL})$, cooled and the precipitate was filtered off. It was dissolved in $150 \mathrm{~mL}$ of trichloroethene with heating and filtered through a layer of silica gel $(3 \mathrm{~cm})$. The solvent was evaporated under reduced pressure and the residue was crystallized from toluene to yield $0.60 \mathrm{~g}$ (76\%) of 2-chloro-3-(1-oxo-3phenylpropynyl)anthraquinone 6b; m.p. $232-233^{\circ} \mathrm{C}$; IR $\left(\mathrm{CHCl}_{3}\right), v_{\max }: 1670,1690(\mathrm{C}=\mathrm{O}), 2205$ $(\mathrm{C} \equiv \mathrm{C}) \mathrm{cm}^{-1} ;{ }^{1} \mathrm{H} \mathrm{NMR}\left(\mathrm{CDCl}_{3}, 200 \mathrm{MHz}\right) \delta 7.40-7.55(\mathrm{~m}, 3 \mathrm{H}, 3 \mathrm{HPh}), 7.65-7.75(\mathrm{~m}, 2 \mathrm{H}, 2 \mathrm{HPh})$, 7.80-7.90 (m, 2H, H-6,7), 8.30-8.40 (m, 2H, H-5,8), 8.40 (s, 1H, H-1(4)), 8.97 (s, 1H, H-4(1)); Anal. Calcd for $\mathrm{C}_{23} \mathrm{H}_{11} \mathrm{O}_{3} \mathrm{Cl}$ : C, 74.50; H, 2.99; Cl, 9.56. Found: C, 74.68; H, 3.09; Cl, 10.06\%.

2-Chloro-3-[3-(1-cyclohexenyl)-1-oxopropynyl]anthraquinone (6f). The experiment was performed by the general procedure with 2-chloroanthraquinonoyl-3 chloride 13 (0.58 g, 1.9 $\mathrm{mmol})$ and 1-ethynylcyclohexene $(0.35 \mathrm{~g}, 3.3 \mathrm{mmol})$. The crude product was isolated and purified as described above for $\mathbf{6 b}$ to give 2-chloro-3-[3-(1-cyclohexenyl)-1oxopropynyl]anthraquinone $6 \mathbf{6 f}(0.35 \mathrm{~g}, 49 \%)$; which was not sufficiently stable, and was used without further purification; ; IR $\left(\mathrm{CHCl}_{3}\right), v_{\max }$ : 1680, $1690(\mathrm{C}=\mathrm{O}), 2230(\mathrm{C} \equiv \mathrm{C})\left(\mathrm{CH}_{2}\right) \mathrm{cm}^{-1} ;{ }^{1} \mathrm{H}$ 
NMR $\left(\mathrm{CDCl}_{3}, 200 \mathrm{MHz}\right) \delta 1.55-1.70\left(\mathrm{~m}, 4 \mathrm{H}, 4^{\prime}, 5^{\prime}-\mathrm{CH}_{2}\right), 2.20-2.35$ (m, 4H, 3', 6'- $\left.\mathrm{CH}_{2}\right), 6.60-$ 6.70 (m, 1H, 2'-CH), 7.80-7.90 (m, 2H, H-6,7), 8.30-8.40 (m, 2H, H-5,8), 8.35 (s, 1H, H-1(4)), 8.85 (s, 1H, H-4(1)).

\section{General procedure for cyclocondensation}

Anthra[1,2-b]thiophene-6,11-dione (2a). To a suspension of $\mathrm{Na}_{2} \mathrm{~S}(0.30 \mathrm{~g}, 3.8 \mathrm{mmol})$ in ethanol $(35 \mathrm{~mL})$ at heating $\left(\approx 60^{\circ} \mathrm{C}\right)$ was added 1-chloro-2-ethynylanthraquinone 1a $(0.30 \mathrm{~g}, 1.1$ $\mathrm{mmol}$ ), then stirred at reflux for $15 \mathrm{~min}$ (monitoring by TLC). The mixture was poured into 300 $\mathrm{mL}$ of water and extracted with $\mathrm{CHCl}_{3}(2 \times 100 \mathrm{~mL})$. The organic layer was washed with water, dried over $\mathrm{MgSO}_{4}$ and evaporated under reduced pressure. The crude product was purified by flash chromatography on silica gel in benzene and crystallized from a toluene-hexane mixture to yield $0.17 \mathrm{~g}(57 \%)$ of anthra[1,2-b]thiophene-6,11-dione 2a; m.p. 216-217 ${ }^{\circ} \mathrm{C} ;{ }^{1} \mathrm{H} \mathrm{NMR}\left(\mathrm{CDCl}_{3}\right.$, $200 \mathrm{MHz}) \delta 7.48(\mathrm{~d}, 1 \mathrm{H}, J=5.5 \mathrm{~Hz}, \mathrm{H}-3), 7.90(\mathrm{~d}, 1 \mathrm{H}, J=5.5 \mathrm{~Hz}, \mathrm{H}-2), 7.75-7.85$ (m, 2H, H8,9), $8.18(\mathrm{~d}, 1 \mathrm{H}, J=8.2 \mathrm{~Hz}, \mathrm{H}-4(5)), 8.30-8.35$ (m, 3H, H-5(4),7,10); Anal. Calcd for $\mathrm{C}_{16} \mathrm{H}_{8} \mathrm{O}_{2} \mathrm{~S}: \mathrm{C}, 72.71 ; \mathrm{H}, 3.05 ; \mathrm{S}, 12.13$. Found: C, 72.52; H, 2.94; S, 11.86\%.

2-Phenylanthra[1,2-b]thiophene-6,11-dione (2b). 1-Chloro-2-phenylethynylanthraquinone 1b $(0.30 \mathrm{~g}, 0.9 \mathrm{mmol})$ was condensed with $\mathrm{Na}_{2} \mathrm{~S}(0.30 \mathrm{~g}, 3.8 \mathrm{mmol})$ in $65 \mathrm{~mL}$ of ethanol for $20 \mathrm{~min}$ by the general procedure. The crude product was purified by flash chromatography on silica gel in $\mathrm{CHCl}_{3}$ and recrystallized from toluene to give $0.27 \mathrm{~g}(90 \%)$ of 2-phenylanthra[1,2b]thiophene-6,11-dione 2b; m.p. $287-288^{\circ} \mathrm{C}$; ${ }^{1} \mathrm{H}$ NMR $\left(\mathrm{CDCl}_{3}, 200 \mathrm{MHz}\right) \delta 7.40-7.55$ (m, 3H, $3 \mathrm{HPh}$ ), 7.65 (s, 1H, H-3), 7.80-7.90 (m, 4H, 2HPh, H-8,9), 8.12 (d, 1H, J = 8.2 Hz, H-4(5)), 8.30-8.40 (m, 3H, H-5(4),7,10); Anal. Calcd for $\mathrm{C}_{22} \mathrm{H}_{12} \mathrm{O}_{2} \mathrm{~S}: \mathrm{C}, 77.63$; H, 3.55; S, 9.42. Found: C, 77.60; H, 3.69; S, 9.40\%.

2-(1-Hydroxy-1-methylethyl)anthra[1,2-b]thiophene-6,11-dione (2c). The experiment was carried out by the general procedure with 1-chloro-2-(3-hydroxy-3-methylbutynyl)anthraquinone 1c $(0.48 \mathrm{~g}, 1.5 \mathrm{mmol})$ and $\mathrm{Na}_{2} \mathrm{~S}(0.48 \mathrm{~g}, 6.1 \mathrm{mmol})$ in $80 \mathrm{~mL}$ of ethanol, the reaction time was 15 min. The crude product was chromatographed on silica gel using $\mathrm{CHCl}_{3}$-ether as the eluent and recrystallized from toluene to yield $0.40 \mathrm{~g}(83 \%)$ of 2-(1-hydroxy-1-methylethyl)anthra[1,2b]thiophene-6,11-dione 2c; m.p. $184-184.5^{\circ} \mathrm{C} ;{ }^{1} \mathrm{H} \mathrm{NMR}\left(\mathrm{CDCl}_{3}, 200 \mathrm{MHz}\right) \delta 1.78$ (s, 6H, Me), 2.30 (brs, 1H, OH), 7.28 (s, 1H, H-3), 7.75-7.85 (m, 2H, H-8,9), 8.01 (d, 1H, J = 8.2 Hz, H4(5)), 8.20-8.35 (m, 3H, H-5(4),7,10); Anal. Calcd for $\mathrm{C}_{19} \mathrm{H}_{14} \mathrm{O}_{3} \mathrm{~S}: \mathrm{C}, 70.79 ; \mathrm{H}, 4.38$; S, 9.95. Found: C, 70.90; H, 4.54; S, 9.91\%.

2-(1-Hydroxymethylpropyl)anthra[1,2-b]thiophene-6,11-dione (2d). The condensation of 1 chloro-2-(3-hydroxy-4-methylpentynyl)anthraquinone 1d $(0.28 \mathrm{~g}, 0.8 \mathrm{mmol})$ with $\mathrm{Na}_{2} \mathrm{~S}(0.30 \mathrm{~g}$, $3.8 \mathrm{mmol}$ ), isolation and purification of the crude product were carried out by the general procedure to give 2-(1-hydroxymethylpropyl)anthra[1,2-b]thiophene-6,11-dione 2d (0.21 g, 75 \%); m.p. $162-163^{\circ} \mathrm{C} ;{ }^{1} \mathrm{H} \mathrm{NMR}\left(\mathrm{CDCl}_{3}, 200 \mathrm{MHz}\right) \delta 0.99(\mathrm{~d}, 3 \mathrm{H}, J=6.7 \mathrm{~Hz}, \mathrm{Me}), 1.07$ (d, 3H, = 6.7 Hz, Me), 2.10-2.25 (m, 1H, $\underline{\mathrm{CHMe}}$ ), 2.30 (brs, $1 \mathrm{H}, \mathrm{OH}), 4.82(\mathrm{~d}, 1 \mathrm{H}, J=4.7 \mathrm{~Hz}, \mathrm{CHO})$, 7.28 (s, 1H, H-3), 7.75-7.85 (m, 2H, H-8,9), 8.03 (d, 1H, J = 8.2 Hz, H-4(5)), 8.25-8.35 (m, 3H, 
H-5(4),7,10); Anal. Calcd for $\mathrm{C}_{20} \mathrm{H}_{16} \mathrm{O}_{3} \mathrm{~S}: \mathrm{C}, 71.41$; H, 4.79; S, 9.53. Found: C, 71.27; H, 4.71; S, $9.38 \%$.

2-Hydroxymethylanthra[1,2-b]thiophene-6,11-dione (2e). The reaction of 1-chloro-2-(3hydroxy- propynyl)anthraquinone 1 e $(0.30 \mathrm{~g}, 1.0 \mathrm{mmol})$ with $\mathrm{Na}_{2} \mathrm{~S}(0.30 \mathrm{~g}, 3.8 \mathrm{mmol})$, isolation, and purification of the crude product were carried out by general procedure to give 2hydroxymethylanthra[1,2-b]thiophene-6,11-dione 2e (0.26 g, 87 \%); m.p. $212-213{ }^{\circ} \mathrm{C} ;{ }^{1} \mathrm{H}$ NMR $\left(\mathrm{CDCl}_{3}, 200 \mathrm{MHz}\right) \delta 2.23$ (brs, 1H, OH), 5.00 (s, 2H, $\left.\mathrm{CH}_{2}\right), 7.33$ (s, 1H, H-3), 7.75-7.85 (m, 2H, $\mathrm{H}-8,9), 8.05(\mathrm{~d}, 1 \mathrm{H}, J=8.2 \mathrm{~Hz}, \mathrm{H}-4(5)), 8.25-8.35$ (m, 3H, H-5(4),7,10); Anal. Calcd for $\mathrm{C}_{17} \mathrm{H}_{10} \mathrm{O}_{3} \mathrm{~S}$ : C, 69.37; H, 3.42; S, 10.89. Found: C, 69.26; H, 3.65; S, 10.81\%.

Anthra[2,1-b]thiophene-6,11-dione (4a). The experiment was carried out by the general procedure with 2-chloro-1-ethynylanthraquinone 3a $(0.17 \mathrm{~g}, 0.6 \mathrm{mmol})$ and $\mathrm{Na}_{2} \mathrm{~S}(0.25 \mathrm{~g}, 3.2$ $\mathrm{mmol}$ ) in $35 \mathrm{~mL}$ of ethanol during $15 \mathrm{~min}$. The crude product was purified by flash chromatography on $\mathrm{Al}_{2} \mathrm{O}_{3}$ in toluene and recrystallized from a toluene-hexane mixture to yield anthra[2,1-b]thiophene-6,11-dione 4a (0.11 g, 66 \%); m.p. 182-183.5 ${ }^{\circ} \mathrm{C} ;{ }^{1} \mathrm{H} \mathrm{NMR}\left(\mathrm{CDCl}_{3}, 200\right.$ MHz) $\delta 7.75-7.85(\mathrm{~m}, 2 \mathrm{H}, \mathrm{H}-8,9), 7.81(\mathrm{~d}, 1 \mathrm{H}, J=5.5 \mathrm{~Hz}, \mathrm{H}-2(1)), 8.20-8.35$ (m, 4H, H4,5,7,10), $8.81(\mathrm{~d}, 1 \mathrm{H}, J=5.5 \mathrm{~Hz}, \mathrm{H}-1(2))$; Anal. Calcd for $\mathrm{C}_{16} \mathrm{H}_{8} \mathrm{O}_{2} \mathrm{~S}: \mathrm{C}, 72.71 ; \mathrm{H}, 3.05 ; \mathrm{S}$, 12.13. Found: C, 72.54; H, 3.01; S, 11.93\%.

2-Phenylanthra[2,1-b]thiophene-6,11-dione (4b). a) 2-Chloro-1-phenylethynylanthraquinone $3 \mathbf{b}^{26}(0.20 \mathrm{~g}, 5.8 \mathrm{mmol})$ was condensed with $\mathrm{Na}_{2} \mathrm{~S}(0.30 \mathrm{~g}, 3.8 \mathrm{mmol})$ in $40 \mathrm{~mL}$ of ethanol for 20 min by the general procedure. The crude product was chromatographed on silica gel using toluene as the eluent and recrystallized from toluene to yield 2-phenylanthra[2,1-b]thiophene6,11-dione 4b (0.17g, 84\%); m.p. 246.5-247.5 ${ }^{\circ} \mathrm{C} ;{ }^{1} \mathrm{H}$ NMR $\left(\mathrm{CDCl}_{3}, 200 \mathrm{MHz}\right) \delta 7.40-7.55$ (m, $3 \mathrm{H}, 3 \mathrm{HPh}$ ), 7.75-7.90 (m, 4H, 2HPh, H-8,9), 8.19 (d, 1H, J = 8.4 Hz, H-4(5)), 8.25-8.35 (m, 3H, $\mathrm{H}-5(4), 7,10), 9.08$ (s, 1H, H-1); Anal. Calcd for $\mathrm{C}_{22} \mathrm{H}_{12} \mathrm{O}_{2} \mathrm{~S}: \mathrm{C}, 77.63$; H, 3.55; S, 9.42. Found: C, 77.67; H, 3.61; S, 9.50\%.

b) The condensation of 1-phenylethynylanthraquinone $9 \mathbf{b}^{26}(0.20 \mathrm{~g}, 0.7 \mathrm{mmol})$ with $\mathrm{Na}_{2} \mathrm{~S}$ $(0.20 \mathrm{~g}, 2.6 \mathrm{mmol})$ in $25 \mathrm{~mL}$ of ethanol under the same conditions afforded $\mathbf{4 b}(0.21 \mathrm{~g}, 95 \%)$.

2-(1-Hydroxy-1-methylethyl)anthra[2,1-b]thiophene-6,11-dione (4c). a) The reaction of 2chloro-1-(3-hydroxy-3-methylbutynyl)anthraquinone 3c $(0.25 \mathrm{~g}, 0.8 \mathrm{mmol})$ with $\mathrm{Na}_{2} \mathrm{~S}(0.30 \mathrm{~g}$, $3.8 \mathrm{mmol}$ ) in $30 \mathrm{ml}$ of ethanol was carried out by the general procedure. The crude product was chromatographed on silica gel in $\mathrm{CHCl}_{3}$ and recrystallized from a toluene-hexane mixture to give 2-(1-hydroxy-1-methylethyl)anthra[2,1-b]thiophene-6,11-dione 4c (0.24 g, 97 \%); m.p. 171-172 ${ }^{\circ} \mathrm{C} ;{ }^{1} \mathrm{H}$ NMR $\left(\mathrm{CDCl}_{3}, 200{ }^{1} \mathrm{H} \mathrm{NMR}\left(\mathrm{CDCl}_{3}, 200 \mathrm{MHz}\right) \delta 1.77\right.$ (s, 6H, Me), 2.32 (brs, $1 \mathrm{H}, \mathrm{OH}), 7.75-7.85$ (m, 2H, H-8,9), 8.14 (d, 1H, J=8.4 Hz, H-4(5)), 8.24 (d, 1H, J = 8.4 Hz, H5(4)), 8.25-8.30 (m, 2H, H-7,10), 8.59 (s, 1H, H-1); Anal. Calcd for $\mathrm{C}_{19} \mathrm{H}_{14} \mathrm{O}_{3} \mathrm{~S}: \mathrm{C}, 70.79$; $\mathrm{H}$, 4.38; S, 9.95. Found: C, 70.80; H, 4.20; S, 9.81\%.

b) 1-(3-Hydroxy-3-methylbutynyl)anthraquinone $9 \mathrm{c}^{29}(0.20 \mathrm{~g}, 0.7 \mathrm{mmol})$ was condensed with $\mathrm{Na}_{2} \mathrm{~S}(0.20 \mathrm{~g}, 2.6 \mathrm{mmol})$ in $25 \mathrm{~mL}$ of ethanol for $15 \mathrm{~min}$ under the same conditions to give $4 \mathrm{c}$ $(0.21 \mathrm{~g}, 95 \%)$. 
2-Phenylanthra[1,2-b]thiopyran-4,7,12-trione (7b). The reaction of 1-chloro-2-(1-oxo-3phenyl- propynyl)anthraquinone $5 \mathbf{b}(0.52 \mathrm{~g}, 1.4 \mathrm{mmol})$ with $\mathrm{Na}_{2} \mathrm{~S}(0.50 \mathrm{~g}, 6.4 \mathrm{mmol})$ in $90 \mathrm{~mL}$ of ethanol was carried out by the general procedure. The crude product was purified by crystallization from a toluene-hexane mixture to yield 2-phenylanthra[1,2-b]thiopyran-4,7,12trione $7 \mathbf{b}\left(0.42\right.$ g, 82 \%); m.p. $291-292^{\circ} \mathrm{C} ;{ }^{1} \mathrm{H} \mathrm{NMR}\left(\mathrm{CDCl}_{3}, 200 \mathrm{MHz}\right) \delta 7.30$ (s, 1H, H-3), 7.50-7.60 (m, 3H, 3HPh), 7.75-7.90 (m, 4H, 2HPh, H-9, 10), 8.25-8.40 (m, 2H, H-8,11), 8.50 (d, $1 \mathrm{H}, J=8.3 \mathrm{~Hz}, \mathrm{H}-5(6)), 9.07$ (d, $1 \mathrm{H}, J=8.3 \mathrm{~Hz}, \mathrm{H}-6(5))$; Anal. Calcd for $\mathrm{C}_{23} \mathrm{H}_{12} \mathrm{O}_{3} \mathrm{~S}: \mathrm{C}, 74.98$; H, 3.28; S, 8.70. Found: C, 75.20; H, 3.43; S, 8.60\%.

2-(1-Hydroxy-1-methylethyl)anthra[1,2-b]thiopyran-4,7,12-trione (7c). 1-Chloro-2-(4hydroxy-4-methyl-1-oxo-pentynyl)anthraquinone 5c $(0.40 \mathrm{~g}, 1.1 \mathrm{mmol})$ was condensed with $\mathrm{Na}_{2} \mathrm{~S}(0.40 \mathrm{~g}, 5.1 \mathrm{mmol})$ in $60 \mathrm{~mL}$ of ethanol for $15 \mathrm{~min}$ by the general procedure. The crude product was purified by flash chromatography on silica gel in $\mathrm{CHCl}_{3}$ and recrystallized from a toluene-hexane mixture to yield 2-(1-hydroxy-1-methylethyl)anthra[1,2-b]thiopyran-4,7,12trione 7c (0.31 g, $78 \%$ \%); m.p. 261-262 ${ }^{\circ} \mathrm{C} ;{ }^{1} \mathrm{H}$ NMR $\left(\mathrm{CDCl}_{3}, 200 \mathrm{MHz}\right) \delta 1.75$ (s, 6H, Me), 2.35 (s, 1H, OH), 7.20 (s, 1H, H-3), 7.80-7.90 (m, 2H, H-9,10), 8.25-8.35 (m, 2H, H-8,11), 8.45 (d, $1 \mathrm{H}, J=8.3 \mathrm{~Hz}, \mathrm{H}-5(6)), 8.99$ (d, $1 \mathrm{H}, J=8.3 \mathrm{~Hz}, \mathrm{H}-6(5))$; Anal. Calcd for $\mathrm{C}_{20} \mathrm{H}_{14} \mathrm{O}_{4} \mathrm{~S}$ : C, 68.56; H, 4.03; S, 9.15. Found: C, 68.51; H, 4.30; S, 9.13\%.

2-(1-Cyclohexenyl)anthra[1,2-b]thiopyran-4,7,12-trione (7f). The condensation of 1-chloro-2[3-(1-cyclohexenyl)-1-oxopropynyl]anthraquinone $5 f(0.40 \mathrm{~g}, 1.1 \mathrm{mmol})$ with $\mathrm{Na}_{2} \mathrm{~S}(0.45 \mathrm{~g}, 5.8$ mmol) was performed by the general procedure. The crude product was purified by crystallization from a toluene-hexane mixture to give 2-(1-cyclohexenyl)anthra[1,2-b]thiopyran4,7,12-trione 7f (0.32 g, $81 \%$ ); m.p. 261-263 ${ }^{\circ} \mathrm{C} ;{ }^{1} \mathrm{H} \mathrm{NMR}\left(\mathrm{CDCl}_{3}, 200 \mathrm{MHz}\right) \delta 1.60-1.90$ (m, 4H, 4', 5'- $\left.\mathrm{CH}_{2}\right), 2.30-2.45$ (m, 4H, 3', 6'- $\left.\mathrm{CH}_{2}\right), 6.75-6.80$ (m, 1H, 2'-CH), 6.98 (s, 1H, H-3), 7.80-7.90 (m, 2H, H-9,10), 8.25-8.35 (m, 2H, H-8,11), 8.40 (d, 1H, J = 8.3 Hz, H-5(6)), 8.94 (d, $1 \mathrm{H}, J=8.3 \mathrm{~Hz}, \mathrm{H}-6(5))$; Anal. Calcd for $\mathrm{C}_{23} \mathrm{H}_{16} \mathrm{O}_{3} \mathrm{~S}$ : C, 74.17; H, 4.33; S, 8.61. Found: C, 74.30; $\mathrm{H}, 4.53 ; \mathrm{S}, 8.40 \%$.

2-Butylanthra[1,2-b]thiopyran-4,7,12-trione (7g). The experiment was performed by the general procedure with 1-chloro-2-(1-oxohept-2-ynyl)anthraquinone $5 \mathrm{~g}(0.50 \mathrm{~g}, 1.4 \mathrm{mmol})$ and $\mathrm{Na}_{2} \mathrm{~S}(0.50 \mathrm{~g}, 6.4 \mathrm{mmol})$. The crude product was chromatographed on silica gel in toluene and crystallized from a toluene-hexane mixture to yield $0.40 \mathrm{~g}(80 \%)$ of 2-butylanthra[1,2b]thiopyran-4,7,12-trione 7g; m.p. $182-183{ }^{\circ} \mathrm{C} ;{ }^{1} \mathrm{H} \mathrm{NMR}\left(\mathrm{CDCl}_{3}, 200 \mathrm{MHz}\right) \delta 0.97$ (t, 3H, J = 7.6 $\mathrm{Hz}, \mathrm{Me}$ ), 1.45 (sextet, $2 \mathrm{H}, J=7.6 \mathrm{~Hz}, \gamma-\mathrm{CH}_{2}$ ), 1.78 (quintet, $2 \mathrm{H}, J=7.6 \mathrm{~Hz}, \beta-\mathrm{CH}_{2}$ ), $2.76(\mathrm{t}, 2 \mathrm{H}$, $\left.J=7.6 \mathrm{~Hz}, \alpha-\mathrm{CH}_{2}\right), 6.94$ (s, 1H, H-3), 7.80-7.90 (m, 2H, H-9,10), 8.25-8.35 (m, 2H, H-8,11), $8.44(\mathrm{~d}, 1 \mathrm{H}, J=8.3 \mathrm{~Hz}, \mathrm{H}-5(6)), 8.99$ (d, $1 \mathrm{H}, J=8.3 \mathrm{~Hz}, \mathrm{H}-6(5))$; Anal. Calcd for $\mathrm{C}_{21} \mathrm{H}_{16} \mathrm{O}_{3} \mathrm{~S}$ : C, 72.39; H, 4.63; S, 9.20. Found: C, 72.50; H, 4.73; S, 9.05\%.

2-(1-Methylprop-1-enyl)anthra[1,2-b]thiopyran-4,7,12-trione (7h). The reaction of a mixture of Z- and E- isomers of 1-chloro-2-(4-methyl-1-oxo-hex-4-en-2-ynyl)anthraquinone $5 \mathbf{h}$ ( $0.40 \mathrm{~g}$, $1.1 \mathrm{mmol})$ with $\mathrm{Na}_{2} \mathrm{~S}(0.40 \mathrm{~g}, 5.1 \mathrm{mmol})$ was run by the general procedure. The crude product was purified by flash chromatography on silica gel in toluene and crystallized from a toluenehexane mixture to yield $0.27 \mathrm{~g}(68 \%)$ of 2-(1-methylprop-1-enyl)anthra[1,2-b]thiopyran-4,7,12- 
trione 7h (a mixture of $Z$ - and E-isomers); m.p. $170-171{ }^{\circ} \mathrm{C} ;{ }^{1} \mathrm{H} \mathrm{NMR}\left(\mathrm{CDCl}_{3}, 200 \mathrm{MHz}\right) \delta 1.72$ (brd, $3 \mathrm{H}, J=7.0 \mathrm{~Hz}, \underline{\mathrm{MeCH}}=\mathrm{C} E$-isomer), 1.92 (brd, $3 \mathrm{H}, J=7.0 \mathrm{~Hz}, \underline{\mathrm{MeCH}}=\mathrm{C} Z$-isomer), 2.10 (brs, $3 \mathrm{H}, \mathrm{CH}_{3}-\mathrm{C}=\mathrm{C}$ ), 5.80 (brq, $1 \mathrm{H}, J=7.0 \mathrm{~Hz}, \mathrm{CH}=\mathrm{C}$ E-isomer), 6.55 (brq, $1 \mathrm{H}, J=7.0 \mathrm{~Hz}$, $\mathrm{CH}=\mathrm{C}$ Z-isomer), 6.87 (s, 1H, H-3 E-isomer), 7.03 (s, 1H, H-3 Z-isomer), 7.80-7.90 (m, 2H, H9,10), 8.25-8.40 (m, 2H, H-8,11), 8.44 (d, 1H, $J=8.3 \mathrm{~Hz}, \mathrm{H}-5(6) \mathrm{Z}$-isomer), 8.48 (d, 1H, $J=8.3$ Hz, H-5(6) E-isomer), 8.99 (d, 1H, $J=8.3 \mathrm{~Hz}, \mathrm{H}-6(5) \mathrm{Z}$-isomer); 9.05 (d, 1H, J=8.3 Hz, H-6(5) E-isomer); Anal. Calcd for $\mathrm{C}_{21} \mathrm{H}_{14} \mathrm{O}_{3} \mathrm{~S}$ : C, 72.81; H, 4.07; S, 9.26. Found: C, 72.85; H, 4.32; S, $9.13 \%$.

2-Phenylanthra[2,3-b]thiopyran-4,6,12-trione (8b). The experiment was carried out by the general procedure with 2-chloro-3-(1-oxo-3-phenylpropynyl)anthraquinone $6 \mathbf{b}(0.30 \mathrm{~g}, 0.8$ $\mathrm{mmol})$ and $\mathrm{Na}_{2} \mathrm{~S}(0.45 \mathrm{~g}, 5.8 \mathrm{mmol})$ in $80 \mathrm{~mL}$ of ethanol. The product was purified by crystallization from toluene to give $0.28 \mathrm{~g}$ (94\%) of 2-phenylanthra[2,3-b]thiopyran-4,6,12trione 8b; m.p.338-339 ${ }^{\circ} \mathrm{C}$; ${ }^{1} \mathrm{H}$ NMR $\left(\mathrm{CDCl}_{3}, 200 \mathrm{MHz}\right) \delta 7.32$ (s, 1H, H-3), 7.50-7.60 (m, 3H, 3HPh), 7.70-7.80 (m, 2H, 2HPh), 7.80-7.90 (m, 2H, H-8,9), 8.35-8.45 (m, 2H, H-7,10), 8.61 (s, 1H, H-5(12)), 9.45 (s, 1H, H-12(5)); Anal. Calcd for $\mathrm{C}_{23} \mathrm{H}_{12} \mathrm{O}_{3} \mathrm{~S}$ : C, 74.98; H, 3.28; S, 8.70. Found: C, 75.05; H, 3.16; S, 8.51\%.

2-(1-Cyclohexenyl)anthra[2,3-b]thiopyran-4,6,12-trione (8f). The experiment was carried out by the general procedure with 2-chloro-3-[3-(1-cyclohexenyl)-1-oxopropynyl]anthraquinone $6 f$ $(0.20 \mathrm{~g}, 0.5 \mathrm{mmol})$ and $\mathrm{Na}_{2} \mathrm{~S}(0.30 \mathrm{~g}, 3.8 \mathrm{mmol})$. The crude product was chromatographed on silica gel in $\mathrm{CHCl}_{3}$ and recrystallized from toluene to yield $0.15 \mathrm{~g}$ (75\%) of 2-(1cyclohexenyl)anthra[2,3-b]thiopyran-4,6,12-trione 8d; m.p. 290-291 ${ }^{\circ} \mathrm{C} ;{ }^{1} \mathrm{H} \mathrm{NMR}\left(\mathrm{CDCl}_{3}, 200\right.$ MHz) $\delta$ 1.60-1.90 (m, 4H, 4', 5'- $\left.\mathrm{CH}_{2}\right), 2.15-2.50$ (m, 4H, 3', 6'- $\left.\mathrm{CH}_{2}\right), 6.65-6.75$ (m, 1H, 2'$\mathrm{CH}), 7.02$ (s, 1H, H-3), 7.80-7.90 (m, 2H, H-8,9), 8.30-8.45 (m, 2H, H-7,10), 8.51 (s, 1H, H5(12)), 9.37 (s, 1H, H-12(5)); Anal. Calcd for $\mathrm{C}_{23} \mathrm{H}_{16} \mathrm{O}_{3} \mathrm{~S}: \mathrm{C}, 74.17$; H, 4.33; S, 8.61. Found: $\mathrm{C}$, 73.68; H, 4.32; S, 8.08\%.

\section{References}

1. Yue, D.; Larock, R.C. J. Org. Chem. 2002, 67, 1905.

2. McDonald, F.E.; Burova, S.A.; Huffman. L.G. Synthesis 2000, 970.

3. Tolstikova, T. G.; Davydova, V. A.; Shul'ts, E. E.; Vafina, G. F.; Safarova, G. M.; Zarudii, F. A.; Lazareva, D. N.; Tolstikov, G. A. Khim.-Farm. Zh. 1990, 24, 27 (Russ.).

4. Kita, Y.; Kirihara, M.; Sekihachi, J.; Okunaka, R.; Sasho, M.; Mohri, Sh.; Honda, T.; Akai, Sh.; Tamura, Y.; Shimooka, K. Chem. Pharm. Bull. 1990, 38, 1836.

5. Ohba, Y.; Murakami, Y.; Sone, T; Awano, H. J. Heterocycl. Chem. 1997, 34, 781.

6. Deng, X.; Liebeskind, L. S. J. Am. Chem. Soc. 2001, 123, 7703.

7. Sall, D.J.; Bastian, J.A.; Briggs, S.L.; Buben, J.A.; Chirgadze, N.Y.; Clawson, D.K.; Denney, M.L.; Giera, D.D.; Gifford-Moore, D.S.; Harper, R.W.; Hauser, K.L.; Klimkowski, V.J.; Kohn, T.J.; Lin, H. Sh.; McCowan, J.R.; Palkowitz, A.D.; Smith, G.F.; Takeuchi, K.; 
Thrasher, K.J.; Tinsley, J.M.; Utterback, B.G., Yan, S.-Ch. B.; Zhang, M. J. Med. Chem. 1997, 40, 3489.

8. Bradley, D.Y., Godfrey, A.G., Schmid, C.R. Tetrahedron Lett. 1999, 40, 5155.

9. Piskunov, A.V.; Shvartsberg, M.S. Mendeleev Commun. 1995, 155.

10. Shvartsberg, M.S.; Ivanchikova, I.D.; Lebedeva, N.I. Tetrahedron Lett. 2000, 41, 5757.

11. Shvartsberg, M.S.; Piskunov, A.V.; Mzhelskaya, M.A.; Moroz, A.A. Izv. Akad. Nauk, Ser. Khim. 1993, 1423; Russ. Chem. Bull. 1993, 42, 1357.

12. Shvartsberg, M.S.; Barabanov, I.I.; Fedenok, L.G. Mendeleev Commun. 1997, 98.

13. Shvartsberg, M.S.; Ivanchikova, I.D.; Vasilevsky, S.F. Tetrahedron Lett. 1994, 35, 2077.

14. Mzhelskaya, M.A.; Moroz, A.A.; Shvartsberg, M.S. Izv. Akad. Nauk Ser. Khim. 1991, 1656; Bull. Acad. Sci. USSR, Div. Chem. Sci. 1991, 40, 1469.

15. Shvartsberg, M.S.; Ivanchikova, I.D.; Fedenok, L.G. Tetrahedron Lett. 1994, 35, 6749.

16. Shvartsberg, M.S.; Ivanchikova, I.D. Tetrahedron Lett. 2000, 41, 771.

17. Barabanov, I.I.; Ivanchikova, I.D.; Shvartsberg, M.S. Mendeleev Commun. 2000, 188.

18. Fedenok, L.G.; Barabanov, I.I.; Ivanchikova, I.D. Tetrahedron 2001, 57, 1331.

19. Larock, C.R.; Yue, D. Tetrahedron Lett. 2001, 42, 6011.

20. Flynn, B.L.; Verdier-Pinard, P.; Hamel, E. Org. Lett. 2001, 5, 651.

21. Malte, A.M.; Castro, C.E. J. Am. Chem. Soc. 1967, 89, 6770.

22. Lendel, V.G.; Pakh, V. J.; Petrus, V.V.; Kijak, M.Yu.; Migalina, Yu.V. Khim. Geterotsikl. Soedin. 1990, 1331 (Russ.).

23. Smirnov-Zamkov I.V.; Zborovsky, Yu.L. Zh. Organ. Khim. 1977, 667 (Russ).

24. Sakamoto, T.; Kondo, Y.; Watanabe, R.; Yamanaka, H. Chem. Pharm. Bull. 1986, 34, 2719.

25. Gorelik, M.V. Chemistry of Anthraquinones and Their Derivatives; Khimiya: Moscow, 1983, pp 296.

26. Piskunov, A.V.; Moroz, A.A.; Shvartsberg, M.S. Izv. Akad. Nauk SSSR, Ser. Khim. 1986, 864; Bull. Acad. Sci. USSR, Div. Chem. Sci. 1986, 35, 785.

27. Hauser, F. M.; Rhee, R. P. J. Org. Chem. 1980, 45, 3061.

28. JP 61/224992. JPN. - Chem. Abstr. 1987, 106, 137013 h.

29. Piskunov, A.V.; Moroz, A.A.; Shvartsberg, M.S. Izv. Akad. Nauk SSSR, Ser. Khim. 1987, 828; Bull. Acad. Sci. USSR, Div. Chem. Sci. 1987, 36, 755.

30. Zanina, A.S.; Shergina, S.I.; Sokolov, I.E.; Kotlyarevsky, I.L Izv. Akad. Nauk SSSR, Ser. Khim. 1981, 1158 (Russ.). 